\title{
Amor y Sexismo: una peligrosa relación en los y las adolescentes gallegos/as
}

\author{
Yolanda Rodríguez Castro*, María Lameiras Fernández*, María Victoria Carrera*
}

Facultad de Ciencias de la Educación, Universidad de Vigo (España)

\begin{abstract}
Resumen
El objetivo de nuestro estudio consiste en analizar la relación entre la visión del amor que presentan los y las adolescentes con las actitudes sexistas ambivalentes hacia mujeres. Para ello, contamos con una muestra representativa a nivel autonómico formada por un total de 800 alumnos/as de $2^{\circ}$ ciclo de la E.S.O. con una media de edad de 15.9 años (DT: 1.50). Se les administró un cuestionario para medir las actitudes, los mitos hacia el amor y las actitudes sexistas. Nuestros resultados evidencian las claras diferencias en relación a la visión que tienen sobre el amor los chicos y las chicas. En cuanto a la relación entre amor y sexismo, destacamos que el sexismo benevolente correlaciona fuertemente con el mito del amor idealizado, amor romántico, amistoso, pragmático, obsesivo y altruista, mientras que sexismo hostil correlaciona con el mito de la vinculación amor-violencia y el amor lúdico.

Palabras clave: amor, sexismo, adolescentes
\end{abstract}

Los mitos y las actitudes hacia el amor constituyen elementos esenciales en el imaginario occidental, y especialmente en la etapa de la adolescencia.

El estudio del amor conoció su mayor apogeo en el campo de la Psicología desde mediados de la década de los sesenta (Sangrador, 1993), se pueden identificar dos líneas de teóricas de investigación: por un lado, las teorías centradas en el desarrollo de la intimidad y por otro, aquellas dirigidas hacia el estudio del amor, que han experimentado un mayor auge y difusión.

$\mathrm{Al}$ indagar en la naturaleza del amor, nos encontramos con la paradoja de la sociedad postmoderna, de individualidad junto a dependencia (Beck \& Beck, 2001). Sin embargo el amor goza de la capacidad universal de permitir trascender a la individualidad y a la separatividad aunque no es una experiencia que todos los sujetos experimentan por igual, de modo que en las diversas formas de vivir y experimentar la vinculación afectiva existen diferentes significados y significantes en función del género (Lameiras, Carrera \& Rodríguez, 2013). Significados y significantes que deben ser explicados en base a los estereotipos de género en los que mujeres y hombres somos socializados, que posibilitan y potencian su vivencia $\mathrm{y}$ expresión diferencial en "femenino" y en "masculino". Estereotipos que no permiten a las mujeres ser ellas mismas ni mantener su integridad, sino que las engulle en una espiral de dominación-sumisión, en la que todo vale, hasta la pérdida de la propia identidad, con la finalidad de obtener el reconocimiento del otro.

En España, los diversos estudios con población adulta se centran en el estudio del amor y en sus falsas creencias (Barrón, Martínez, De Paul \& Yela, 1999; Ferrer, Boch \& Navarro, 2010). Los resultados de estos estudios confirman la existencia de diferencias en las concepciones sobre amor entre mujeres y hombres. De manera que en gran medida, las mujeres siguen creyendo que tenemos una pareja predestinada y que con el amor se puede superar cualquier problema u obstáculo en la relación (Barrón et al., 1999; Ferrer et al., 2010); mientras que los hombres siguen concediendo importancia al hecho de tener pareja (Ferrer et al., 2010). Estos aspectos diferenciales de la socialización del amor en femenino y en masculino pueden observarse desde la infancia. Las mujeres, desde muy pequeñas, aprenden a amar para la "fusión", interiorizando una ética del cuidado que les relega al ámbito doméstico y reproductivo, abocándoles a la desvaloración de sí mismas como sujetos autónomos, que sólo pueden ser y existir a través de la entrega abnegada al otro (Lameiras, Carrera \& Rodríguez, 2009). Por otra parte, los hombres interiorizan desde pequeños su rol en el espacio público, orientado a la competitividad y al poder, totalmente alejados de las emociones, se les enseña a vincularse desde y para la "separación", única forma de obtención de su identidad como sujeto autónomo (Lameiras et al., 2009). Estas diferencias están fuertemente vinculadas con los estereotipos de género y con las asimetrías de poder que hombres y mujeres tienen en la sociedad actual (Maltas, 2003).

Para poder profundizar y avanzar en el estudio del amor como medida de prevención de la violencia en las relaciones de pareja de los y las jóvenes es necesario contar con estudios capaces de identificar las creencias erróneas hacia el amor que asumen los y las adolescentes sino también las actitudes sexistas. Para la comprensión de la nueva cara del sexismo la aportación más significativa y novedosa es la teoría del sexismo ambivalente de Glick y Fiske (1996), que es la primera que reconoce la necesidad de ubicar en la comprensión del nuevo sexismo la dimensión relacional. El sexismo ambivalente se operativiza con la presencia de dos elementos con cargas afectivas antagónicas: positivas y negativas (Glick \& Fiske, 1996). Dando lugar a dos tipos de sexismo vinculados: sexismo hostil y sexismo benevolente. El sexismo hostil ( $\mathrm{SH}$ ) es una ideología que caracteriza a las mujeres como un grupo subordinado e inferior y legitima el control social que ejercen los hombres. Por su parte, el sexismo benevolente (SB) se basa en una ideología tradicional que idealiza a las mujeres como esposas, madres y objetos románticos (Glick et al., 1996).

En este sentido, las ideas asociadas al amor y los modelos amorosos imperantes en nuestra sociedad están intrínsecamente unidos a la violencia de género (Flecha, Puigvert \& Redondo, 2005). Es por tanto, el objetivo de este estudio analizar la relación entre la visión del amor que presentan los y las adolescentes con las actitudes sexistas ambivalentes hacia mujeres. 


\section{Método}

\section{Participantes}

Contamos con una muestra representativa a nivel autonómico formada por un total de 800 alumnos y alumnas del segundo ciclo de la Educación Secundaria Obligatoria de las cuatro provincias de Galicia. La edad media es de 15.9 años (DT: 1.50) y el rango de edad oscila entre los 14 y los 20 años.

\section{Instrumentos}

Se administró un cuestionario que incluye información relativa al género, la edad, a si tenían o habían tenido pareja así como el tiempo de la relación, y las siguientes escalas:

Love Attitudes Scale (LAS) (Hendrick et al., 1998), se empleó la versión breve validada en España de Rodríguez-Castro, Lameiras, Carrera, Vallejo-Medina (2013a). Se tratan de 18 ítems que miden las actitudes hacia seis estilos de amor que se evalúan mediante tres ítems cada uno: Eros o Amor Pasional; Ludus o Amor Lúdico; Storge o Amor amistoso; Pragma o Amor Pragmático; Manía o Amor Obsesivo; y, finalmente, Ágape o Amor Altruista con un formato de respuesta de esta escala es de tipo Likert: 1 (totalmente en desacuerdo) a 5 (totalmente de acuerdo). Mayores puntuaciones indican actitudes más positivas hacia el estilo a evaluar. En el presente estudio la fiabilidad oscila entre .68 y .88 .

Escala de Mitos hacia el Amor (Bosch et al. 2007). Se ha empleado la adaptación de la escala para adolescentes (Rodríguez, Lameiras, Carrera \& Vallejo-Medina, 2013b) formada por 7 ítems que evaluarían seis mitos ya mencionados y distribuidos en dos factores: el primer factor denominado Mito de la idealización del amor (ítems 1, 2, 3, 4 y 5,); y el segundo factor denominado Mito de la vinculación amor-maltrato (ítems 6 y 7). El formato de respuesta de tipo Likert (1 totalmente en desacuerdo a 5 totalmente de acuerdo). El alfa obtenido en nuestro estudio fue en el factor 1 fue de $0,70 \mathrm{y}$ en el factor 2 de 0,86 .

Escala de Sexismo Ambivalente (ASI), de Glick y Fiske (1996) en la versión española reducida de Rodríguez, Lameiras \& Carrera (2009) con la que se miden las actitudes sexistas hostiles (tradicionales) y benevolentes (tono afectivo positivo) hacia las mujeres. El rango de respuesta es de tipo Likert que va de 0 (totalmente en desacuerdo) a 5 (totalmente de acuerdo). Las mayores puntuaciones indican mayor nivel de sexismo hacia las mujeres. Las fiabilidades obtenidas en nuestro estudio fueron de 0.81 en SH y 0.76 en SB.

\section{Procedimiento}

Una vez seleccionados los centros educativos realizamos su captación, remitiendo al director/a del centro seleccionado una carta oficial del organismo y personas responsables del estudio. Posteriormente se contactó con los centros telefónicamente para confirmar su colaboración, fijando la fecha para aplicar el cuestionario a al estudiantado. Los cuestionarios siendo anónimos y voluntarios, fueron aplicados en horario lectivo y disponían aproximadamente de una hora para cubrir los cuestionarios.

\section{Resultados}

Para comprobar las diferencias entre chicos y chicas en relación a las creencias asociadas al amor tomamos inicialmente los dos factores de la escala de Mitos hacia el amor (ver tabla 1). En el mito de amor idealizado no se detectan diferencias significativas en función al género aunque si se observa la tendencia de las chicas a estar más de acuerdo con esta visión idealizada del amor. Para profundizar un poco más en esta tendencia, se decidió analizar de forma separada los mitos que conforman el mito del amor idealizado, encontrando diferencias significativas en dos de los mitos (el mito de pasión eterna y el mito de la omnipotencia). De forma que son las chicas las que estarían más de acuerdo con el mito de la pasión eterna y el mito de la omnipotencia. En cuanto al mito de la vinculación amor-maltrato encontramos diferencias significativas en función del género, siendo los chicos los que están más de acuerdo con este mito. En relación a las actitudes hacia el amor, son los chicos los que muestran una visión más lúdica y altruista del amor que sus compañeras. En relación a las actitudes sexistas, los chicos muestran mayor nivel de sexismo hostil que las chicas y presentar un alto nivel de sexismo benevolente al igual que sus compañeras.

Tabla 1. Diferencias de medias en función al género, en relación a las creencias asociadas al amor, a las actitudes hacia el amor y las actitudes sexistas ambivalentes.

\begin{tabular}{|c|c|c|c|}
\hline & $\begin{array}{c}\text { M (DT) } \\
\text { Chicos }\end{array}$ & $\begin{array}{c}\text { M } \\
\text { (DT) } \\
\text { Chicas }\end{array}$ & t-Student \\
\hline Mito Amor Idealizado & $\begin{array}{c}17.9 \\
(4.28)\end{array}$ & $\begin{array}{c}18.5 \\
(4.01)\end{array}$ & -1.54 \\
\hline M. Media Naranja & $\begin{array}{c}3.50 \\
(1.34)\end{array}$ & $\begin{array}{c}3.51 \\
(1.37)\end{array}$ & -.129 \\
\hline M. Pasión Eterna & $\begin{array}{c}4.08 \\
(1.06)\end{array}$ & $\begin{array}{c}4.30 \\
(1.00)\end{array}$ & $-2.14^{*}$ \\
\hline M. Omnipotencia & $\begin{array}{c}3.41 \\
(1.28)\end{array}$ & $\begin{array}{c}3.88 \\
(1.22)\end{array}$ & $-3.79 * * *$ \\
\hline M. de los Celos & $\begin{array}{c}3.00 \\
(1.32)\end{array}$ & $\begin{array}{c}2.83 \\
(1.39)\end{array}$ & 1.23 \\
\hline Mito Amor Maltrato & $\begin{array}{c}3.14 \\
(1.95)\end{array}$ & $\begin{array}{c}2.61 \\
(1.44)\end{array}$ & $3.09 * *$ \\
\hline Amor amistoso & $\begin{array}{c}1.7 \\
(.45)\end{array}$ & $\begin{array}{l}1.65 \\
(.47)\end{array}$ & 1.25 \\
\hline Amor lúdico & $\begin{array}{l}1.51 \\
(.50)\end{array}$ & $\begin{array}{l}1.33 \\
(.47)\end{array}$ & $3.76 * * *$ \\
\hline Amor romántico & $\begin{array}{l}1.91 \\
(.28)\end{array}$ & $\begin{array}{l}1.91 \\
(.28)\end{array}$ & .136 \\
\hline Amor pragmático & $\begin{array}{l}1.51 \\
(.50)\end{array}$ & $\begin{array}{l}1.58 \\
(.49)\end{array}$ & -1.38 \\
\hline Amor obsesivo & $\begin{array}{l}1.71 \\
(.44)\end{array}$ & $\begin{array}{l}1.69 \\
(.46)\end{array}$ & 1.04 \\
\hline Amor Altruista & $\begin{array}{l}1.83 \\
(.36)\end{array}$ & $\begin{array}{l}1.72 \\
(.44)\end{array}$ & $2.86 * *$ \\
\hline Sexismo hostil & $\begin{array}{l}20.2 \\
(6.7)\end{array}$ & $\begin{array}{l}15.0 \\
(5.5)\end{array}$ & $8.35 * * *$ \\
\hline Sexismo benevolente & $\begin{array}{l}21.2 \\
(6.8)\end{array}$ & $\begin{array}{l}20.3 \\
(7.1)\end{array}$ & 1.33 \\
\hline
\end{tabular}


Tal y como era de esperar, se observa como el sexismo ambivalente están relacionados con los mitos del amor y las actitudes hacia el amor (ver Tabla 2). Por un lado el sexismo benevolente está fuertemente relacionado con el mito del amor ideal mientras el sexismo hostil correlaciona más débilmente. De forma que los chicos y las chicas con actitudes sexistas benevolentes son los que tienen visiones más idealizadas del amor. En cuanto al mito de la vinculación amor-maltrato sólo correlaciona moderadamente con el sexismo hostil. En este sentido, los chicos y las chicas más sexistas hostiles son los que en mayor medida aceptan la vinculación del amor con la violencia. En cuanto a las actitudes hacia el amor que muestran los y las adolescentes, los chicos y las chicas más sexistas hostiles son los que tienen una visión más lúdica del amor. Sin embargo, los chicos y las chicas que muestran mayores niveles de sexismo benevolentes son los que tienen una visión del amor más romántica, amistosas, pragmática, obsesiva y altruista.

Tabla 2. Correlaciones de Pearson entre los mitos del amor. Actitudes hacia el amor con las subescalas de sexismo ambivalente (SH y SB)

\begin{tabular}{lcc} 
& SH & SB \\
\hline $\begin{array}{l}\text { Mito amor } \\
\text { idealizado }\end{array}$ & $0.15^{* *}$ & $0.41^{* *}$ \\
Mito amor maltrato & $0.30^{* *}$ & 0.09 \\
& & \\
Romántico & .01 & $.22^{* *}$ \\
Lúdico & $.12^{*}$ & -.01 \\
Amistoso & .01 & $.16^{* *}$ \\
Pragmático & .05 & $.11^{* *}$ \\
Obsesivo & .06 & $.24^{* *}$ \\
Altruista & .07 & $.26^{* *}$ \\
\hline ota. ${ }^{* *} \mathrm{p}<0,01 ; * \mathrm{p}<0,05 ; \mathrm{SH}=$ sexismo hostil; SB $=$
\end{tabular}

Nota. ${ }^{* *} \mathrm{p}<0,01 ;{ }^{*} \mathrm{p}<0,05 ; \mathrm{SH}=$ sexismo hostil; $\mathrm{SB}=$ sexismo benevolente.

\section{Discusión}

En relación a los resultados obtenidos sobre las actitudes y creencias asociadas al amor, encontramos en nuestro estudio que los y las adolescentes asumen diferentes concepciones sobre el amor, ya que las chicas son las que presentan una visión más idealizada del amor, a pesar que las diferencias no son significativas, sí que se aprecia la tendencia de ellas a estar más de acuerdo con las creencias asociados a la existencia de que tenemos una pareja predestinada en nuestra vida, que el amor romántico y pasional puede perdurar a través del tiempo, y que cuando existe amor en una pareja, el amor es tan poderoso que sirve para vencer cualquier obstáculo o problema que pueda surgir en una relación. Por otra parte también son los chicos los que presentan una visión del amor más lúdica y altruista. Además, esta visión del amor idealizada del amor, romántica, amistosa pragmática, obsesiva y alturista está fuertemente relacionada con la cara más dulce del sexismo hacia las mujeres que es el benevolente, que con su manto afectivo positivo encubre una visión estereotipada de la mujer, que no la considera inferior al hombre pero si diferente y por ello, es necesario cuidarla, proveerla y protegerla (Glick \& Fiske, 1996; Lameiras et al., 2013).

Los chicos sí que presentan de una forma más latente su mayor aceptación al mito del amor maltratador frente a sus compañeras. Además se obtiene una fuerte vinculación con la cara más dura del sexismo que es el sexismo hostil. De forma que los chicos y las chicas más sexistas hostiles son los presentan puntuaciones más elevadas en el mito del amor maltratador. Diversos estudios han evidenciado que el sexismo hostil es un importante predictor de las actitudes a favor de la violencia contra las mujeres (Rodríguez, Magalhaes \& Peixoto, 2010); mientras que el sexismo benevolente asume su vertiente protectora hacia la mujer que acepta su rol tradicional de esposa y madre (Allen, Swan \& Raghavan, 2009)

En este sentido, las palabras de Marcela Lagarde (2005) sobre el amor están muy en sintonía con nuestros resultados, ya que el amor, tan central en la vida de las mujeres, resulta el espacio más tradicional en las mujeres modernas, y al mismo tiempo, se convierte en el espacio más vulnerable ya que construimos nuestra identidad en base a un yo relacional, de cuidado y de entrega hacia la otra persona. Por ello, es importante como una medida de prevención frente a la violencia contra las mujeres, saber situar el amor en la vida de la mujer y al mismo tiempo, saber situarse en el amor que requiere un trabajo de crecimiento personal para no confundir el amor con otras cosas: la posesión, la opresión, la anulación (Sanz, 1995). Por lo tanto, se hace imprescindible llevar a cabo una reformulación del amor (Boch, Ferrer \& Alzamora, 2006), donde las partes implicadas estén en igualdad de condiciones y que asuman como propios valores como el respeto, la confianza, el compromiso emocional, y la reciprocidad. Así desde la comunidad educativa, se deben llevar a cabo programas de prevención de la violencia en las relaciones de pareja de los y las adolescentes en los que trabaje en profundidad el mundo de los afectos así como los estereotipos de género y las actitudes sexistas.

\section{Referencias}

Allen, C., Swan, S. \& Raghavan, C. (2009). Gender Symmetry, Sexism, and Intimate Partner Violence. Journal of Interpersonal Violence, 24/11, 1816-1834.

Beck, U. \& Beck, E. (2001). El normal caos del Amor: las nuevas formas de relación amorosa. Barcelona: Paidós Ibérica.

Barrón, A., Martínez, D., De Paul, P. \& Yela, C. (1999). Romantic beliefs and myths in Spain. The Spanish Journal of Psychology, 2, 64-73.

Bosch, E., Ferrer, M. V., García, M. E., Ramis, M. C., Mas, M. C., Navarro, C. \& Torrens, G. (2007). Del mito del amor romántico a la violencia contra las mujeres en la pareja. Madrid: Instituto de la Mujer.

Bosch, E., Ferrer, V. \& Alzamora, A. (2006). El laberinto patriarcal. Barcelona: Antrophos. 
Ferrer, V., Bosch, E. \& Navarro, C. (2010). Los mitos románticos en España. Boletín de psicología, 9, 7-31.

Flecha, A., Puigvert, L. \& Redondo, G. (2005). Socialización preventiva de la violencia de género. Feminismos, 6, 107-120.

Glick, P. \& Fiske, S. (1996). The Ambivalent Sexism Inventory: Differentiating hostile and benevolent sexism. Journal of Personality and Social Psychology, 70, 491-512.

Hendrick, C., Hendrick, S. S. \& Dicke, A. (1998). The Love Attitudes Scale: Short form. Journal of Social and Personal Relationships, 15, 147-159.

Lagarde, M. (2005). Para mis socias de la vida. Madrid: Horas y Horas.

Lameiras, M., Carrera, M. V. \& Rodríguez, Y. (2013). Sexualidad y Salud. El estudio de la sexualidad humana desde la perspectiva de género. Vigo: Servicio Publicaciones Universidad de Vigo.

Lameiras, M., Carrera, M. V. \& Rodríguez, Y. (2009). Violencia de Genero: Ideología Patriarcal y actitudes sexistas. En I. Iglesias Canle y M. Lameiras Fernández (Coord.), Violencia de género: Perspectiva jurídica y psicosocial (pp. 117-151), Valencia: Tirant lo blanch.

Maltas, G. (2003). El lastre del adultismo; en O. Guash y O. Viñuales (eds.), Sexualidades. Diversidad y control social (pp. 191-213). Barcelona: Bellaterra.
Rodríguez, Y., Lameiras, M. \& Carrera, M. V. (2009). Validación de la versión reducida de las escalas ASI y AMI en una muestra de estudiantes españoles. Psicogente, 12/2, 284-295.

Rodríguez, Y., Magalhaes, M. J. \& Peixoto, J. M. (2010). Sexismo ambivalente: actitudes y creencias hacia violencia de género. Revista Ártemix, 11, 133-139.

Rodríguez-Castro, Y., Lameiras, M., Carrera, M. V. \& Vallejo, P. (2013a). Validación de la Escala de Actitudes hacia el Amor en una muestra de adolescentes. Revista de Estudios de Psicología, 34(2), 209-219.

Rodríguez-Castro, Y., Lameiras, M., Carrera, M. V. \& Vallejo, P. (2013b). La fiabilidad y validez de la escala de mitos hacia el amor: las creencias de los y las adolescentes. Revista de Psicología Social, 28(2), 157-168.

Sangrador, J. L. (1993). Consideraciones psicosociales sobre el amor romántico. Piscothema, 5, 181-196.

Sanz, F. (1995). Los Vínculos Amorosos. Amor desde la identidad en la terapia de reencuentro. Barcelona: Kairós. 